\title{
A hidden symmetry in quantum gravity
}

\author{
Sudarshan Ananth, ${ }^{a}$ Lars Brink ${ }^{b, c}$ and Sucheta Majumdar ${ }^{a}$ \\ ${ }^{a}$ Indian Institute of Science Education and Research, \\ Pune 411008, India \\ ${ }^{b}$ Department of Physics, Chalmers University of Technology, \\ S-41296 Göteborg, Sweden \\ ${ }^{c}$ Division of Physics and Applied Physics, School of Physical and Mathematical Sciences, \\ Nanyang Technological University, 637371 Singapore \\ E-mail: ananth@iiserpune.ac.in, lars.brink@chalmers.se, \\ sucheta.majumdar@ulb.ac.be
}

ABSTRACT: The action integral contains more information than the equations of motion. Since it is an integral, changes of the integration variables occasionally also expose symmetries more easily than working directly with the equations of motion. We have previously shown that there are signs of an extended exceptional symmetry for $\mathcal{N}=8$ supergravity in four dimensions. The symmetry is such that the fields used in the Lagrangian are not representations of the symmetry. Instead one has to add representations to obtain a representation of the extended symmetry group. In this paper we discuss an extended symmetry in four-dimensional gravity which is the "Ehlers Symmetry" in three dimensions. It cannot be spanned by the helicity states of four-dimensional gravity but it can be realised once we treat the helicity states just as field variables of the functional integral, which can be changed like variables in any integral. We also explain how this symmetry is inherent in formulations of $\mathcal{N}=8$ supergravity in four dimensions through a truncation in the field space to pure gravity. The establishment of these "hidden" symmetries should play an important role in the perturbative behaviour of the quantum theories. Since the method used $n$ this paper is purely algebraic we will not provide any information on the geometric role of these symmetries.

KEywords: Classical Theories of Gravity, Space-Time Symmetries, Supergravity Models ArXiv EPrint: 1808.02498 


\section{Contents}

1 Introduction 1

$2 \mathrm{SU}(1,1)$ in pure gravity in three dimensions 3

2.1 Gravity, in $d=4$, in the light-cone gauge 3

2.2 Gravity, in $d=3$, in the light-cone gauge 4

2.3 The $\mathrm{SU}(1,1)$ symmetry in $d=3 \quad 5$

3 Maximal supergravity in $d=4 \quad 7$

4 Truncation: from supergravity to pure gravity in $d=4 \quad 8$

4.1 Maximal supergravity in three dimensions 9

$\begin{array}{lll}4.2 \text { A lift back to four dimensions } & 10\end{array}$

5 Conclusions 11

\section{Introduction}

Supergravity theories show remarkable quantum properties in the sense that their perturbative expansions are finite to higher loop orders than naïvely expected [1]. Even though we expect all these theories to diverge at some loop order, it is important to understand why this is so. These phenomena must have some root in superstring theory and we expect that the study of the limiting supergravity theories will help us understand superstring theory better. When we study classical gravity in the flat limit, we look for symmetries the theory exhibits in terms of the helicity +2 and -2 fields. In the quantum case, we should study the functional integral over the action where those components are field variables that we integrate over. In the functional integral we can modify them forgetting that they are helicity fields. Hence we can ask ourselves if the functional integral has additional symmetries, over and above the spacetime symmetries that we know.

In this paper, we demonstrate signs of such a hidden symmetry in four-dimensional gravity. We use the light-cone gauge formulation in which the action is an infinite series of higher order terms and we work only up to the four-point level. Accordingly, we cannot prove that the symmetry is a symmetry of the full theory, but our experience from previous work is that if the symmetry works to this order, it is most likely to survive as a symmetry of the full theory (although we will not be able to prove this to all orders within the current formalism). There are also strong indications that a symmetry like this should be anomaly free since the $E_{7}$ of maximally supersymmetric Supergravity is [2]. This is why we stress its importance for the quantum theory, since every symmetry constrains the quantum properties and we need that to explain the "better than expected" quantum properties of the scattering amplitudes. 
This symmetry first appeared in the work of Cremmer and Julia [3], who found an unexpected symmetry at the level of the equations of motion in $N=8$ supergravity. We have shown that in our approach [4] this is indeed a symmetry of the full Hamiltonian and is, in some sense, on an equal footing with the maximal supersymmetry in the theory. ${ }^{1}$

In more recent work we have argued that the $E_{7(7)}$ symmetry should also be present in the original $d=11$ supergravity theory [5]. We then showed that the corresponding $E_{8(8)}$ symmetry, thought to be special to maximal supergravity in $d=3$, could be lifted to a symmetry of the $d=4$ theory and in principle also to the $d=11$ theory [6]. In all these extraordinary cases we claim that the action should exhibit the symmetry. We have to carefully choose combinations of the representations used in a particular dimension to represent the symmetry but the actions do not distinguish between these.

We should remind ourselves that these exceptional symmetries have an origin in the Superstring Theory in the U-duality. We certainly hope to come back to issues we discuss in this paper and our previous ones in the context of the Superstring Theory. For the moment we have nothing to say there but we believe it will be an important study.

A key feature in our analysis is that the Hamiltonians in the maximally supersymmetric cases can be written as quadratic forms [7-9]. However we have also shown that for the non-supersymmetric cases, ie. pure Yang-Mills and pure gravity, this remains the case. In this paper we will investigate possible extra global symmetries in pure $d=4$ gravity.

Our light-cone formulation uses only the physical degrees of freedom. This approach is particularly well suited to the study of symmetries that are not manifest in covariant formulations $[10,11]$. That means that even part of the Poincaré symmetry is non-linearly realised. All remaining symmetries are global and the exceptional ones are described as non-linear $\sigma$-model symmetries. In the case of $E_{7(7)}$ the quotient $E_{7(7)} / \mathrm{SU}(8)$ is non-linearly realised while the $\mathrm{SU}(8)$ is the linear $R$-symmetry. The drawback with this formalism is that we loose the contact with the geometry underlying the theory. We have reduced the problem to a purely algebraic one.

In four-dimensional gravity no such symmetry is known but in three-dimensional gravity there is the "Ehlers symmetry" [12], which is an extra $\operatorname{SL}(2, R)$ symmetry not connected to any space-time symmetry. To find the four-dimensional $\sigma$-model action with this symmetry, one must find a non-trivial change of variables in $d=4$ in the light-cone action. Here we will present an alternative method to find the $\sigma$-model, where we first study the Ehlers symmetry in $d=3$ and realize this symmetry in the four-dimensional action by means of a suitable "oxidation" procedure.

Hence we will first study $d=3$ gravity in the light-cone gauge formulation and show that there is indeed an $\mathrm{SU}(1,1) \sigma$-model symmetry. We will show that this symmetry is easily recognized only after a field redefinition. This is again a manifestation of the change of integration variables, permitted in the functional integral.

The formulation we are using is not easy to lift to $d=4$. However in a recent paper, we treated a similar problem for maximal supergravity. We found a $d=3$ formulation of the theory such that the $E_{8(8)}$ symmetry (in three dimensions) could be carefully "oxidized" to

\footnotetext{
${ }^{1}$ This is because we can also use this symmetry to pin down the possible interaction terms in the Hamiltonian.
} 
four dimensions [6]. We now use that analysis and truncate the superfield until it contains only the gravity degrees of freedom. Indeed, the $E_{8(8)}$ symmetry then reduces to an $\mathrm{SU}(1,1)$ symmetry. The formulation of pure gravity in this manner is probably one of the most impenetrable formulations of ordinary gravity and we do not recommend it for any explicit calculations but it serves its purpose, to show us the hidden symmetry.

\section{$2 \mathrm{SU}(1,1)$ in pure gravity in three dimensions}

In this section, we describe gravity in $d=3$ in the light-cone gauge. We do this by a straightforward dimensional reduction from $d=4$ where the light-cone formulation of gravity is well known [13-15]. After the reduction, we perform a suitable field redefinition that makes the Ehlers symmetry easy to write down.

\subsection{Gravity, in $d=4$, in the light-cone gauge}

With the metric $(-,+,+,+)$, we define the light-cone coordinates

$$
x^{ \pm}=\frac{1}{\sqrt{2}}\left(x^{0} \pm x^{3}\right) ; \quad x=\frac{1}{\sqrt{2}}\left(x_{1}+i x_{2}\right) ; \quad \bar{x}=\frac{1}{\sqrt{2}}\left(x_{1}-i x_{2}\right),
$$

with the corresponding derivatives being $\partial_{ \pm}, \partial$ and $\bar{\partial}$. The Einstein-Hilbert action on a Minkowski background reads

$$
S_{E H}=\int d^{4} x \mathcal{L}=\frac{1}{2 \kappa^{2}} \int d^{4} x \sqrt{-g} \mathcal{R} .
$$

In the light-cone gauge, the Lagrangian density in terms of the helicity states $h$ and $\bar{h}$ to order $\kappa^{2}$ reads [7]

$$
\begin{aligned}
\mathcal{L}= & \frac{1}{2} \bar{h} \square h+2 \kappa \bar{h} \partial^{+^{2}}\left[-h \frac{\bar{\partial}^{2}}{\partial^{+2}} h+\frac{\bar{\partial}}{\partial^{+}} h \frac{\bar{\partial}}{\partial^{+}} h\right]+2 \kappa h \partial^{+2}\left[-\bar{h} \frac{\partial^{2}}{\partial^{+2}} \bar{h}+\frac{\partial}{\partial^{+}} \bar{h} \frac{\partial}{\partial^{+}} \bar{h}\right] \\
& +\frac{1}{\partial^{+2}}\left[\partial^{+} h \partial^{+} \bar{h}\right] \frac{\partial \bar{\partial}}{\partial^{+2}}\left[\partial^{+} h \partial^{+} \bar{h}\right]+\frac{1}{\partial^{+3}}\left[\partial^{+} h \partial^{+} \bar{h}\right]\left(\partial \bar{\partial} h \partial^{+} \bar{h}+\partial^{+} h \partial \bar{\partial} \bar{h}\right) \\
& -\frac{1}{\partial^{+2}}\left[\partial^{+} h \partial^{+} \bar{h}\right]\left(2 \partial \bar{\partial} h \bar{h}+2 h \partial \bar{\partial} \bar{h}+9 \bar{\partial} h \partial \bar{h}+\partial h \bar{\partial} \bar{h}-\frac{\partial \bar{\partial}}{\partial^{+}} h \partial^{+} \bar{h}-\partial^{+} h \frac{\partial \bar{\partial}}{\partial^{+}} \bar{h}\right) \\
& -2 \frac{1}{\partial^{+}}\left[2 \bar{\partial} h \partial^{+} \bar{h}+h \partial^{+} \bar{\partial} \bar{h}-\partial^{+} \bar{\partial} h \bar{h}\right] h \partial \bar{h}-2 \frac{1}{\partial^{+}}\left[2 \partial^{+} h \partial \bar{h}+\partial^{+} \partial h \bar{h}-h \partial^{+} \partial \bar{h}\right] \bar{\partial} h \bar{h} \\
& -\frac{1}{\partial^{+}}\left[2 \bar{\partial} h \partial^{+} \bar{h}+h \partial^{+} \bar{\partial} \bar{h}-\partial^{+} \bar{\partial} h \bar{h}\right] \frac{1}{\partial^{+}}\left[2 \partial^{+} h \partial \bar{h}+\partial^{+} \partial h \bar{h}-h \partial^{+} \partial \bar{h}\right] \\
& -h \bar{h}\left(\partial \bar{\partial} h \bar{h}+h \partial \bar{\partial} \bar{h}+2 \bar{\partial} h \partial \bar{h}+3 \frac{\partial \bar{\partial}}{\partial^{+}} h \partial^{+} \bar{h}+3 \partial^{+} h \frac{\partial \bar{\partial}}{\partial^{+}} \bar{h}\right) .
\end{aligned}
$$

The d'Alembertian in the equation above is $\square=2\left(\partial \bar{\partial}-\partial^{+} \partial^{-}\right)$. The Hamiltonian to order $\kappa^{2}$, corresponding to the Lagrangian above can be be written in the following compact form [9]

$$
\mathcal{H}=\int d^{3} x \mathcal{D} \bar{h} \overline{\mathcal{D}} h
$$

with

$$
\mathcal{D} \bar{h}=\partial \bar{h}+2 \kappa \frac{1}{\partial^{+^{2}}}\left(\frac{\bar{\partial}}{\partial^{+}} h \partial^{+^{3}} \bar{h}-h \partial^{+^{2}} \bar{\partial} \bar{h}\right)+\mathcal{O}\left(\kappa^{2}\right) .
$$

$\overline{\mathcal{D}} h$ is the complex conjugate of the expression above. 


\subsection{Gravity, in $d=3$, in the light-cone gauge}

We dimensionally reduce the pure gravity Lagrangian from $d=4$ to $d=3$ by setting $\partial=\bar{\partial}$

$$
\begin{aligned}
L & =\frac{1}{2} \bar{h} \square h+2 \kappa \bar{h} \partial^{+^{2}}\left(\frac{\partial}{\partial^{+}} h \frac{\partial}{\partial^{+}} h-h \frac{\partial^{2}}{\partial^{+2}} h\right)+\text { c.c. }+O\left(\kappa^{2}\right), \\
& =\mathcal{L}_{0}+\mathcal{L}_{\kappa}+\mathcal{L}_{\kappa^{2}}
\end{aligned}
$$

where the d'Alembertian is now $\square=2\left(\partial^{2}-\partial^{+} \partial^{-}\right)$. This expression is not suitable to search for the Ehlers symmetry. The non-linear part of it should be implemented to lowest order by $\delta h=$ constant + quadratic in field ..., and the three-point coupling is evidently not invariant under such a transformation. In order to find the symmetry we therefore start by eliminating the cubic interaction vertices. We perform the following field redefinitions

$$
h \rightarrow h^{\prime}-\kappa \partial^{+^{2}}\left(\frac{1}{\partial^{+}} h^{\prime} \frac{1}{\partial^{+}} h^{\prime}\right)-2 \kappa \frac{1}{\partial^{+^{2}}}\left(\partial^{+^{3}} h^{\prime} \frac{1}{\partial^{+}} \bar{h}^{\prime}\right),
$$

with a conjugate expression for $\bar{h}$. These field redefinitions eliminate all cubic interaction vertices but introduce the following new quartic vertices into the Lagrangian.

$$
\begin{aligned}
& \kappa^{2}\left\{\partial^{+^{2}}\left(\frac{1}{\partial^{+}} \bar{h} \frac{1}{\partial^{+}} \bar{h}\right)+\frac{1}{\partial^{+2}}\left(\partial^{+3} \bar{h} \frac{1}{\partial^{+}} h\right)\right\} \\
& \times\left(\partial^{2}-\partial^{+} \partial^{-}\right)\left\{\partial^{+^{2}}\left(\frac{1}{\partial^{+}} h \frac{1}{\partial^{+}} h\right)+2 \frac{1}{\partial^{+2}}\left(\partial^{+3} h \frac{1}{\partial^{+}} \bar{h}\right)\right\} \\
& -2 \kappa^{2}\left\{\partial^{+^{2}}\left(\frac{1}{\partial^{+}} \bar{h} \frac{1}{\partial^{+}} \bar{h}\right)+\frac{1}{\partial^{+2}}\left(\partial^{+3} \bar{h} \frac{1}{\partial^{+}} h\right)\right\} \partial^{+^{2}}\left(\frac{\partial}{\partial^{+}} h \frac{\partial}{\partial^{+}} h-h \frac{\partial^{2}}{\partial^{+2}} h\right) \\
& -4 \kappa^{2} \bar{h} \partial^{+^{2}}\left[\frac{\partial}{\partial^{+}}\left\{\partial^{+^{2}}\left(\frac{1}{\partial^{+}} h \frac{1}{\partial^{+}} h\right)+2 \frac{1}{\partial^{+2}}\left(\partial^{+3} h \frac{1}{\partial^{+}} \bar{h}\right)\right\} \frac{\partial}{\partial^{+}} h\right] \\
& +2 \kappa^{2} \bar{h} \partial^{+^{2}}\left[\left\{\partial^{+^{2}}\left(\frac{1}{\partial^{+}} h \frac{1}{\partial^{+}} h\right)+2 \frac{1}{\partial^{+2}}\left(\partial^{+^{3}} h \frac{1}{\partial^{+}} \bar{h}\right)\right\} \frac{\partial^{2}}{\partial^{+2}} h\right] \\
& -2 \kappa^{2} \bar{h} \partial^{+^{2}}\left[h \frac{\partial^{2}}{\partial^{+2}}\left\{\partial^{+^{2}}\left(\frac{1}{\partial^{+}} h \frac{1}{\partial^{+}} h\right)+2 \frac{1}{\partial^{+2}}\left(\partial^{+^{3}} h \frac{1}{\partial^{+}} \bar{h}\right)\right\}\right] .
\end{aligned}
$$

The first two lines in the expression above involve $\partial^{-}$, which are time derivatives, and hence need to be eliminated. This is achieved by adding terms of order $\kappa^{2}$ to the field redefinition (2.7) which now reads

$$
\begin{aligned}
h \rightarrow & h^{\prime}-\kappa \partial^{+^{2}}\left(\frac{1}{\partial^{+}} h^{\prime} \frac{1}{\partial^{+}} h^{\prime}\right)-2 \kappa \frac{1}{\partial^{+2}}\left(\partial^{+3} h^{\prime} \frac{1}{\partial^{+}} \bar{h}^{\prime}\right) \\
& +\kappa^{2} \frac{1}{\partial^{+2}}\left\{\frac{1}{\partial^{+}} \bar{h}^{\prime} \partial^{+5}\left(\frac{1}{\partial^{+}} h^{\prime} \frac{1}{\partial^{+}} h^{\prime}\right)\right\}+4 \kappa^{2} \frac{1}{\partial^{+2}}\left\{\partial^{+}\left(\partial^{+3} h^{\prime} \frac{1}{\partial^{+}} \bar{h}^{\prime}\right) \frac{1}{\partial^{+}} \bar{h}^{\prime}\right\} \\
& +2 \kappa^{2} \frac{1}{\partial^{+2}}\left\{\partial^{+3} h^{\prime} \frac{1}{\partial^{+3}}\left(\partial^{+3} \bar{h}^{\prime} \frac{1}{\partial^{+}} h^{\prime}\right)\right\}+2 \kappa^{2} \partial^{+^{2}}\left\{\frac{1}{\partial^{+3}}\left(\partial^{+3} h^{\prime} \frac{1}{\partial^{+}} \bar{h}^{\prime}\right) \frac{1}{\partial^{+}} h^{\prime}\right\} .
\end{aligned}
$$

We thus arrive at a $d=3$ Lagrangian in the following form

$$
\mathcal{L}^{\prime}=\mathcal{L}_{0}+\mathcal{L}^{\prime}{ }^{2}
$$


where the new quartic interaction Lagrangian is

$$
\begin{aligned}
\mathcal{L}^{\prime}{ }_{\kappa^{2}}= & \mathcal{L}_{\kappa^{2}}-\kappa^{2} \partial^{+4}\left(\frac{1}{\partial^{+}} \bar{h} \frac{1}{\partial^{+}} \bar{h}\right)\left[\frac{\partial}{\partial^{+}} h \frac{\partial}{\partial^{+}} h-h \frac{\partial^{2}}{\partial^{+2}} h\right] \\
& +4 \kappa^{2} \partial^{+3} \bar{h} \frac{1}{\partial^{+}} h\left[\frac{\partial}{\partial^{+}} h \frac{\partial}{\partial^{+}} h-h \frac{\partial^{2}}{\partial^{+2}} h\right]-2 \kappa^{2} \frac{1}{\partial^{+4}}\left(\partial^{+3} h \frac{1}{\partial^{+}} \bar{h}\right) \partial^{+4} \bar{h} \frac{\partial^{2}}{\partial^{+2}} h \\
& +4 \kappa^{2} \frac{1}{\partial^{+4}}\left(\partial^{+3} h \frac{1}{\partial^{+}} \bar{h}\right) \partial^{+3} \partial \bar{h} \frac{\partial}{\partial^{+}} h-2 \kappa^{2} \frac{1}{\partial^{+4}}\left(\partial^{+3} h \frac{1}{\partial^{+}} \bar{h}\right) h \partial^{+2} \partial^{2} \bar{h} \\
& -8 \kappa^{2} \partial^{+^{2}} \bar{h} \frac{\partial}{\partial^{+2}}\left(\partial^{+3} h \frac{1}{\partial^{+}} \bar{h}\right) \frac{\partial}{\partial^{+}} h+2 \kappa^{2} \partial^{+^{2}} \bar{h} \frac{1}{\partial^{+2}}\left(\partial^{+3} h \frac{1}{\partial^{+}} \bar{h}\right) \frac{\partial^{2}}{\partial^{+2}} h \\
& +4 \kappa^{2} \partial^{+2} \bar{h} h \frac{\partial^{2}}{\partial^{+4}}\left(\partial^{+3} h \frac{1}{\partial^{+}} \bar{h}\right),
\end{aligned}
$$

with the first term denoting the old quartic interaction Lagrangian.

\subsection{The $\mathrm{SU}(1,1)$ symmetry in $d=3$}

The Hamiltonian (to order $\kappa^{2}$ ) corresponding to (2.10) is

$$
\mathcal{H}=\bar{h} \partial^{2} h-\mathcal{L}^{\prime}{ }^{2} .
$$

We can now ask if this expression could be invariant under a $\sigma$-model like symmetry of the schematic form

$$
\delta h=\text { constant }+h h+h h h h+\ldots,
$$

where we have not distinguished between $h$ and $\bar{h}$. This is quite straightforward to check and indeed we find it is invariant under the following transformations (to order $\kappa$ )

$$
\delta h=\frac{1}{\kappa} a-\kappa a \frac{1}{\partial^{+}}\left(\partial^{+} h \bar{h}\right)-2 \kappa a \frac{1}{\partial^{+2}}\left(\partial^{+3} h \frac{1}{\partial^{+}} \bar{h}\right)-2 \kappa \bar{a} \frac{1}{\partial^{+^{2}}}\left(\partial^{+3} h \frac{1}{\partial^{+}} \bar{h}\right)+\frac{1}{2} \kappa \bar{a} h h,
$$

and

$$
\delta \bar{h}=\frac{1}{\kappa} \bar{a}-\kappa a \frac{1}{\partial^{+}}\left(\partial^{+} \bar{h} h\right)-2 \kappa a \frac{1}{\partial^{+^{2}}}\left(\partial^{+3} \bar{h} \frac{1}{\partial^{+}} h\right)-2 \kappa a \frac{1}{\partial^{+2}}\left(\partial^{+^{3}} \bar{h} \frac{1}{\partial^{+}} h\right)+\frac{1}{2} \kappa a \bar{h} \bar{h} .
$$

The commutator of two such transformations on $h($ or $\bar{h})$ is

$$
\left[\delta_{1}, \delta_{2}\right] h=2\left(\bar{a}_{1} a_{2}-\bar{a}_{2} a_{1}\right) h ; \quad\left[\delta_{1}, \delta_{2}\right] \bar{h}=-2\left(\bar{a}_{1} a_{2}-\bar{a}_{2} a_{1}\right) \bar{h} .
$$

We can rewrite (2.14) and (2.15) as two sets of transformations with parameters $a$ and $\bar{a}$ as follows.

$$
\begin{aligned}
& L_{+} h=\frac{1}{\kappa} a-\kappa a \frac{1}{\partial^{+}}\left(\partial^{+} h \bar{h}\right)-2 \kappa a \frac{1}{\partial^{+2}}\left(\partial^{+3} h \frac{1}{\partial^{+}} \bar{h}\right), \\
& L_{+} \bar{h}=-2 \kappa a \frac{1}{\partial^{+2}}\left(\partial^{+3} \bar{h} \frac{1}{\partial^{+}} h\right)+\frac{1}{2} \kappa a \bar{h} \bar{h} .
\end{aligned}
$$

and

$$
\begin{aligned}
& L_{-} h=-2 \kappa \bar{a} \frac{1}{\partial^{+2}}\left(\partial^{+3} h \frac{1}{\partial^{+}} \bar{h}\right)+\frac{1}{2} \kappa \bar{a} h h, \\
& L_{-} \bar{h}=\frac{1}{\kappa} \bar{a}-\kappa a \frac{1}{\partial^{+}}\left(\partial^{+} \bar{h} h\right)-2 \kappa a \frac{1}{\partial^{+^{2}}}\left(\partial^{+3} \bar{h} \frac{1}{\partial^{+}} h\right) .
\end{aligned}
$$


We define the following $\mathrm{U}(1)$ transformation

$$
L_{0} h=\bar{a} a h ; \quad L_{0} \bar{h}=-\bar{a} a \bar{h} .
$$

These transformations now satisfy an $\mathrm{SU}(1,1)$ algebra

$$
\left[L_{+}, L_{-}\right]=L_{0} ; \quad\left[L_{0}, L_{ \pm}\right]= \pm L_{ \pm} .
$$

This is the light-cone realization of the Ehlers symmetry of General Relativity. The form of the Hamiltonian used here is however not suitable to "oxidize" to four-dimensions. This is most directly done using the Hamiltonian written as a quadratic form as in (2.4) and instead of trying to rewrite the Hamiltonian in such a form (which takes a lot of guesswork and partial integrations to find the final form) we will use another path.

The schematic below explains how the Ehlers symmetry in three-dimensional gravity can be derived from the exceptional symmetry in maximal supergravity and subsequently lifted to four dimensions.
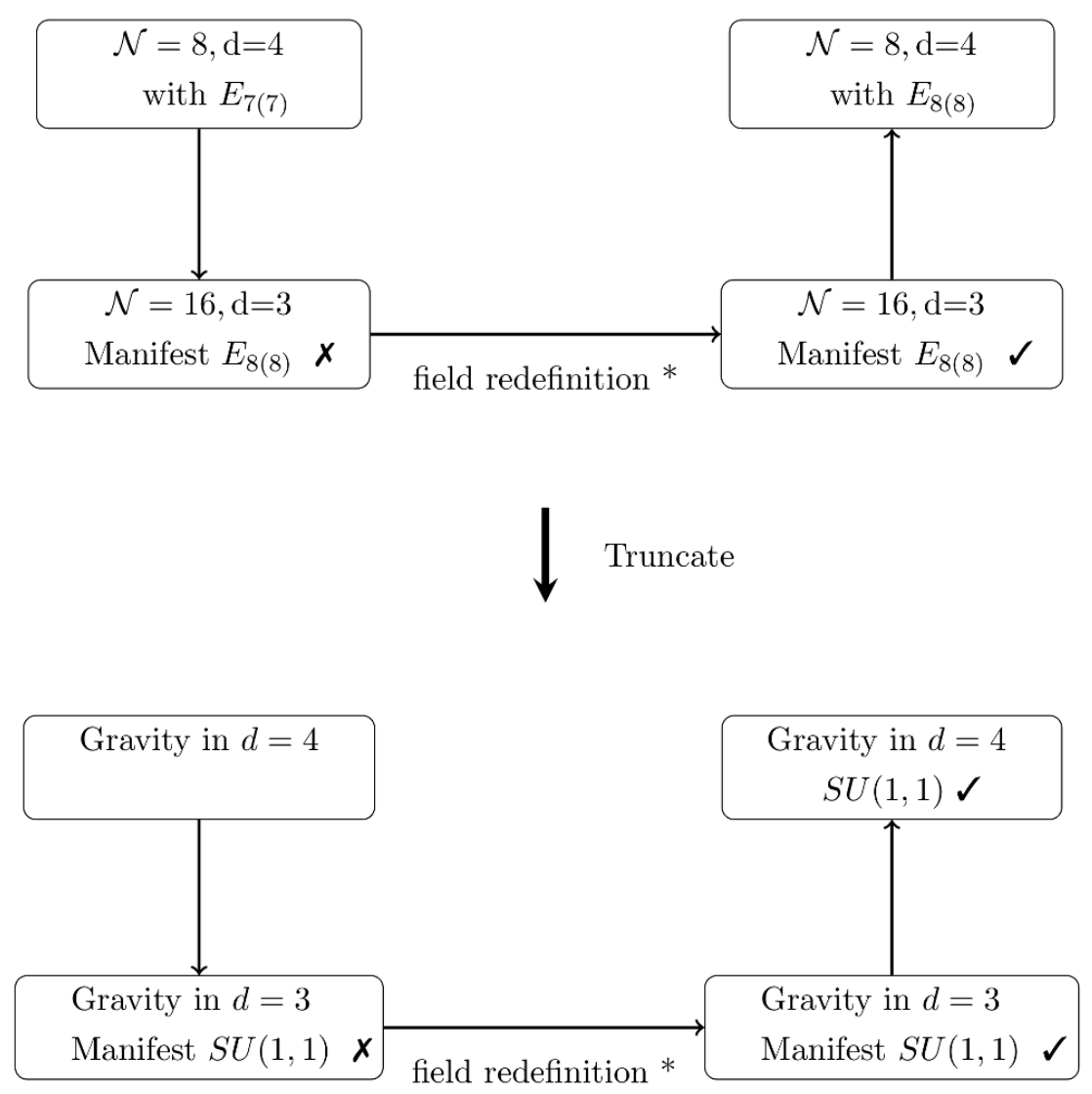

* This field redefinition eliminates cubic vertices in the action.

A suitable truncation of the oxidation procedure adopted in the supergravity case will help us realize the $\mathrm{SU}(1,1)$ symmetry in $d=4$. In order to do this, we briefly present the essential points from our earlier analysis of the $\mathcal{N}=8$ model $[7,9]$. 


\section{Maximal supergravity in $d=4$}

The $\mathcal{N}=8$ supergravity theory in the light-cone gauge formulation is written in a $\mathcal{N}=8$ superspace, spanned by Grassmann variables $\theta^{m}$ and $\bar{\theta}_{m}, m=1 \ldots 8$ ( 8 and $\overline{\mathbf{8}}$ of SU(8)), where all 256 physical degrees of freedom are captured in a single $\mathcal{N}=8$ superfield [16]

$$
\begin{aligned}
\phi(y)= & \frac{1}{\partial^{+2}} h(y)+i \theta^{m} \frac{1}{\partial^{+2}} \bar{\psi}_{m}(y)+\frac{i}{2} \theta^{m} \theta^{n} \frac{1}{\partial^{+}} \bar{A}_{m n}(y), \\
& -\frac{1}{3 !} \theta^{m} \theta^{n} \theta^{p} \frac{1}{\partial^{+}} \bar{\chi}_{m n p}(y)-\frac{1}{4 !} \theta^{m} \theta^{n} \theta^{p} \theta^{q} \bar{C}_{m n p q}(y), \\
& +\frac{i}{5 !} \theta^{m} \theta^{n} \theta^{p} \theta^{q} \theta^{r} \epsilon_{m n p q r s t u} \chi^{s t u}(y), \\
& +\frac{i}{6 !} \theta^{m} \theta^{n} \theta^{p} \theta^{q} \theta^{r} \theta^{s} \epsilon_{\text {mnpqrstu }} \partial^{+} A^{t u}(y), \\
& +\frac{1}{7 !} \theta^{m} \theta^{n} \theta^{p} \theta^{q} \theta^{r} \theta^{s} \theta^{t} \epsilon_{\text {mnpqrstu }} \partial^{+} \psi^{u}(y), \\
& +\frac{4}{8 !} \theta^{m} \theta^{n} \theta^{p} \theta^{q} \theta^{r} \theta^{s} \theta^{t} \theta^{u} \epsilon_{\text {mnpqrstu }} \partial^{+2} \bar{h}(y),
\end{aligned}
$$

with $h$ and $\bar{h}$ representing the graviton, $\bar{\psi}_{m}$ the 8 spin- $\frac{3}{2}$ gravitinos, $\bar{A}_{m n}$ the 28 gauge fields, $\bar{\chi}_{m n p}$ the 56 gauginos and $\bar{C}_{m n p q}$ the 70 real scalars. All fields are local in

$$
y=\left(x, \bar{x}, x^{+}, y^{-} \equiv x^{-}-\frac{i}{\sqrt{2}} \theta^{m} \bar{\theta}_{m}\right) .
$$

Chiral derivatives in this space read

$$
d^{m}=-\frac{\partial}{\partial \bar{\theta}_{m}}-\frac{i}{\sqrt{2}} \theta^{m} \partial^{+} ; \quad \bar{d}_{n}=\frac{\partial}{\partial \theta^{n}}+\frac{i}{\sqrt{2}} \bar{\theta}_{n} \partial^{+}
$$

and the kinematical (spectrum generating) supersymmetry generators are

$$
q_{+}^{m}=-\frac{\partial}{\partial \bar{\theta}_{m}}+\frac{i}{\sqrt{2}} \theta^{m} \partial^{+} ; \quad \bar{q}_{+n}=\frac{\partial}{\partial \theta^{n}}-\frac{i}{\sqrt{2}} \bar{\theta}_{n} \partial^{+} .
$$

To order $\kappa$, the action for $\mathcal{N}=8$ supergravity reads [16]

$$
\begin{gathered}
-\frac{1}{64} \int d^{4} x \int d^{8} \theta d^{8} \bar{\theta} \mathcal{L} \\
\mathcal{L}=-\bar{\phi} \frac{\square}{\partial^{+4}} \phi+\frac{4}{3} \kappa\left(\frac{1}{\partial^{+4}} \bar{\phi} \bar{\partial} \bar{\partial} \phi \partial^{+^{2}} \phi-\frac{1}{\partial^{+4}} \bar{\phi} \partial^{+} \bar{\partial} \phi \partial^{+} \bar{\partial} \phi+\text { c.c. }\right) .
\end{gathered}
$$

Grassmann integration is normalized such that $\int d^{8} \theta(\theta)^{8}=1$. The Hamiltonian for the $\mathcal{N}=8$ theory to order $\kappa^{2}$ can be expressed as a Quadratic Form [7]

$$
\mathcal{H}=\frac{1}{4 \sqrt{2}}\left(\mathcal{W}_{m}, \mathcal{W}_{m}\right)
$$

where the inner product is defined as

$$
(\phi, \xi) \equiv-2 i \int d^{4} x d^{8} \theta d^{8} \bar{\theta} \bar{\phi} \frac{1}{\partial^{+^{3}}} \xi
$$


We note that this is unrelated to the fact that the Hamiltonian is the anticommutator of two supersymmetries. At order $\kappa$,

$$
\begin{aligned}
& \mathcal{W}_{m}=-\frac{\partial}{\partial^{+}} \bar{q}_{+m} \phi-\kappa \frac{1}{\partial^{+}}\left(\bar{\partial} \bar{d}_{m} \phi \partial^{+^{2}} \phi-\partial^{+} \bar{d}_{m} \phi \partial^{+} \bar{\partial} \phi\right)+\mathcal{O}\left(\kappa^{2}\right), \\
& \overline{\mathcal{W}}^{m}=-\frac{\bar{\partial}}{\partial^{+}} q_{+}^{m} \bar{\phi}-\kappa \frac{1}{\partial^{+}}\left(\partial d^{m} \bar{\phi} \partial^{+^{2}} \bar{\phi}-\partial^{+} d^{m} \bar{\phi} \partial^{+} \partial \bar{\phi}\right)+\mathcal{O}\left(\kappa^{2}\right) .
\end{aligned}
$$

$\mathcal{W}_{m}$ at order $\kappa^{2}$ is presented in [4].

The $E_{7(7)} / \mathrm{SU}(8)$ transformation of the $\mathcal{N}=8$ supergravity theory can be written in a compact way by introducing a coherent state-like representation

$$
\delta \phi=-\frac{2}{\kappa} \theta^{i j k l} \bar{\Xi}_{i j k l}+\left.\frac{\kappa}{4 !} \Xi^{i j k l}\left(\frac{\partial}{\partial \eta}\right)_{i j k l} \frac{1}{\partial^{+2}}\left(e^{\eta \hat{\bar{d}}} \partial^{+3} \phi e^{-\eta \hat{\bar{d}}} \partial^{+3} \phi\right)\right|_{\eta=0}+\mathcal{O}\left(\kappa^{3}\right),
$$

where

$$
\begin{gathered}
\theta^{a_{1} a_{2} \ldots a_{n}}=\frac{1}{n !} \theta^{a_{1}} \theta^{a_{2} \cdots} \theta^{a_{n}}, \\
\eta \hat{\bar{d}}=\eta^{m} \frac{\bar{d}_{m}}{\partial^{+}} \text {and }\left(\frac{\partial}{\partial \eta}\right)_{i j k l} \equiv \frac{\partial}{\partial \eta^{i}} \frac{\partial}{\partial \eta^{j}} \frac{\partial}{\partial \eta^{k}} \frac{\partial}{\partial \eta^{l}} .
\end{gathered}
$$

We note that these $E_{7(7)} / \mathrm{SU}(8)$ transformations do close properly to an $\mathrm{SU}(8)$ transformation on the superfield.

\section{Truncation: from supergravity to pure gravity in $d=4$}

We now note that we could set all fields, except $h$ and $\bar{h}$, in the superfield to zero. The resulting expression from (3.6) is then a Hamiltonian describing pure gravity in four dimensions, in the light-cone gauge. This is another way of understanding the result in (2.4).

In other words, the following "superfield"

$$
\phi(y)=\frac{1}{\partial^{+^{2}}} h(y)+\frac{4}{8 !} \theta^{m} \theta^{n} \theta^{p} \theta^{q} \theta^{r} \theta^{s} \theta^{t} \theta^{u} \epsilon_{m n p q r s t u} \partial^{+2} \bar{h}(y),
$$

furnishes us with an unnecessarily complicated description of pure gravity through the Quadratic Form defined by (3.6), (3.8) and (3.9).

We point out that this complicated way of writing gravity was already hinted at by earlier results. In particular, we found that the light-cone Hamiltonians of both pure gravity and maximal supergravity exhibit a quadratic form structure [7, 9]. Earlier, we showed that both pure Yang-Mills theory and the maximally supersymmetric $\mathcal{N}=4$ Yang-Mills also exhibit this quadratic form structure [8]. On the other hand, theories with less-thanmaximal supersymmetry do not possess this property.

This form of the Hamiltonian is not suitable to look for a $\sigma$-model symmetry of the four-dimensional theory. When we truncate the superfield to the gravity case we see that the symmetry (3.10) disappears. In order to find a remnant of an exceptional symmetry in $d=4$ we have again to first dimensionally reduce the $N=8$ theory to three dimensions, make a field redefinition and then lift the theory back to $d=4$ and finally perform the truncation again or make the truncation already in three dimension and then lift it. The two procedures commute. 


\subsection{Maximal supergravity in three dimensions}

In section 3 , we arrived at a description of $d=3$ gravity by dimensional reduction of the component Lagrangian for gravity, in the light-cone gauge. We now have a second path to the same result. When we dimensionally reduce the $d=4$ maximal Supergravity theory theory to $d=3$, we are left with the dependence on one transverse derivative, $\partial$. We obtain, for the action for the $d=3$ theory (up to an overall constant)

$$
\mathcal{S}=\int d^{3} x d^{8} \theta d^{8} \bar{\theta} \mathcal{L}
$$

where

$$
\mathcal{L}=-\bar{\phi} \frac{\square}{\partial^{+^{4}}} \phi+\frac{4}{3} \kappa\left(\frac{1}{\partial^{+4}} \bar{\phi} \partial^{2} \phi \partial^{+^{2}} \phi-\frac{1}{\partial^{+4}} \bar{\phi} \partial^{+} \partial \phi \partial^{+} \partial \phi+\text { c.c. }\right),
$$

This theory does not show an $E_{8(8)}$ symmetry since the $\mathrm{SO}(16) R$-symmetry which is the maximal subgroup of $E_{8(8)}$ and linearly realized does not admit vertices of odd order $\left(\kappa, \kappa^{3}\right.$ etc.). It is spanned on the spinor representation $\mathbf{1 2 8}$ for both the bosons and the fermions and there is no $\mathbf{1}$ in the multiplication of an odd number of such spinor representations. Again we have to make field redefinitions to get rid of the three-point couplings. This was done in [6]. We were again led to a Hamiltonian in a quadratic form

$$
\mathcal{H}^{(3)}=\frac{1}{4 \sqrt{2}}\left(\mathcal{W}^{(3)}{ }_{m}, \mathcal{W}^{(3)}{ }_{m}\right)
$$

with the superscript reminding us that we are working in $d=3$. We could now again truncate the superfield to only contain $h$ and $\bar{h}$ and will then recover the gravity theory in the field representation with no three-point coupling. In [17] the $E_{8,8} / \mathrm{SO}(16)$ transformations were derived. They read

$$
\begin{aligned}
\delta_{E_{8(8)} / \mathrm{SO}(16)} \phi= & \frac{1}{\kappa} F+\kappa \epsilon^{m_{1} m_{2} \ldots m_{8}} \sum_{c=-2}^{2}\left(\hat{\bar{d}}_{m_{1} m_{2} \cdots m_{2(c+2)}} \partial^{+c} F\right) \\
& \times\left\{\left.\left(\frac{\delta}{\delta \eta}\right)_{m_{2 c+5} \cdots m_{8}} \partial^{+(c-2)}\left(e^{\eta \cdot \hat{d}} \partial^{+(3-c)} \phi e^{-\eta \cdot \hat{d}} \partial^{+(3-c)} \phi\right)\right|_{\eta=0}+\mathcal{O}\left(\kappa^{2}\right)\right\},
\end{aligned}
$$

where the sum is over the $\mathrm{U}(1)$ charges $c=2,1,0-1,-2$ of the bosonic fields, and

$$
\begin{aligned}
F= & \frac{1}{\partial^{+2}} \beta\left(y^{-}\right)+i \theta^{m n} \frac{1}{\partial^{+}} \bar{\beta}_{m n}\left(y^{-}\right)-\theta^{m n p q} \bar{\beta}_{m n p q}\left(y^{-}\right) \\
& +i \widetilde{\theta}{ }_{m n} \partial^{+} \beta^{m n}\left(y^{-}\right)+4 \widetilde{\theta} \partial^{+2} \bar{\beta}\left(y^{-}\right), \\
\hat{\bar{d}}_{m_{1} m_{2} \cdots m_{2(c+2)}} \equiv & \hat{\bar{d}}_{m_{1}} \hat{\bar{d}}_{m_{2}} \cdots \hat{\bar{d}}_{2(c+2)}
\end{aligned}
$$

and

$$
\widetilde{\theta}_{a_{1} a_{2} \ldots a_{n}}=\epsilon_{a_{1} a_{2} \ldots a_{n} b_{1} b_{2} \ldots b_{(8-n)}} \theta^{b_{1} b_{2} \cdots b_{(8-n)}} .
$$


$F$ represents the 128 transformation parameters. We now set all the parameters in $F$, other than $\beta$ and $\bar{\beta}$, to zero following (4.1).

$$
F=\frac{1}{\partial^{+^{2}}} \beta\left(y^{-}\right)+4 \widetilde{\theta} \partial^{+^{2}} \bar{\beta}\left(y^{-}\right)
$$

We can then check that the exceptional $E_{8(8)} / \mathrm{SO}(16)$ transformations (4.5) break down to the $L_{-}$and $L_{+}$transformations in section 2 , where the parameters, $a$ and $\bar{a}$ are identified with $\beta$ and $\bar{\beta}$ repectively. Similarly, the $\mathrm{SO}(16)$ breaks down to a $\mathrm{U}(1)$ given by $L_{0}$. In section 2 , we have made this realization entirely explicit.

Having established that the $d=3$ pure gravity theory possesses this symmetry, the natural next step is to ask whether we can oxidize back to four dimensions, exactly as we did with supergravity [6]. Indeed, this can be done as explained below.

\subsection{A lift back to four dimensions}

The result in (4.4) is a particularly powerful way of realizing the Ehlers-symmetry from section 2. This particular form of the Hamiltonian can now be oxidized back to four dimensions, while preserving this Ehlers symmetry.

This is achieved very easily by replacing all the $\partial\left(=\partial_{1}\right)$ by the generalized derivative

$$
\nabla \equiv \partial_{1}+i \partial_{2}
$$

in the expression for $\mathcal{W}_{m}^{(3)}$ to order- $\kappa^{2}[17]$

$$
\begin{aligned}
\epsilon^{m} \mathcal{W}_{m}^{(3)}= & \epsilon^{m} \frac{\partial}{\partial^{+}} \bar{q}_{m} \phi \\
& +\frac{\kappa^{2}}{2} \sum_{c=-2}^{2} \frac{1}{\partial^{+(c+4)}}\left\{\left.\frac{\delta}{\delta a} \frac{\delta}{\delta b}\left(\frac{\delta}{\delta \eta}\right)_{m_{1} m_{2} \ldots m_{2(c+2)}}\left(E \partial^{+(c+5)} \phi E^{-1}\right)\right|_{a=b=\eta=0}\right. \\
& \left.\times\left.\frac{\epsilon^{m_{1} m_{2} \ldots m_{8}}}{(4-2 c) !}\left(\frac{\delta}{\delta \eta}\right)_{m_{2 c+5} \ldots m_{8}} \partial^{+2 c}\left(E \partial^{+(4-c)} \phi E^{-1} \partial^{+(4-c)} \phi\right)\right|_{\eta=0}\right\},
\end{aligned}
$$

where

$$
E \equiv e^{a \hat{\partial}+b \epsilon \hat{q}+\eta \hat{d}} \text { and } E^{-1} \equiv e^{-a \hat{\partial}-b \epsilon \hat{\bar{q}}-\eta \hat{\bar{d}}}
$$

with

$$
a \hat{\partial}=a \frac{\partial}{\partial^{+}}, \quad b \epsilon \hat{\bar{q}}=b \epsilon^{m} \frac{\bar{q}_{m}}{\partial^{+}}, \quad \eta \hat{\bar{d}}=\eta^{m} \frac{\bar{d}_{m}}{\partial^{+}} .
$$

The conjugate derivative, in four dimensions, enters through $\overline{\mathcal{W}}^{(3)}$. The key point is that the $E_{8(8)}$ transformations on $\mathcal{W}^{(3)}$ and $\overline{\mathcal{W}}^{(3)}$ are zero separately. This is why we could argue that also the four-dimensional action has an $E_{8(8)}$ invariance. The same argument goes through in the truncated case. This is then the statement that we find a $\mathrm{SU}(1,1)$ internal symmetry in the $d=4$ light-cone description of the pure gravity action. 


\section{Conclusions}

We have in this paper worked with the action for gravity. It has been natural to ask if it also contains symmetries that are not obvious from or manifest at the level of the equations of motion $[10,11]$. We have shown here that such symmetries do appear in both maximally supersymmetric quantum field theories and pure gravity. The Ehlers symmetry is a wellknown symmetry in three dimensional spacetime. By writing the $d=3$ Hamiltonian in a special manner we have found a way to lift that Hamiltonian to four dimensions while still exhibiting the same symmetry (which is unrelated to spacetime symmetry). In the process, we have written the Hamiltonian in several different ways seemingly getting more and more complicated but in the end finding a form that allows us to uncover this symmetry. We might in the process have found the most round-about and complex way to write the pure gravity Hamiltonian but we are not intending to use this particular form for practical calculations. The symmetries should be present even when we do not explicitly see them and hence affect calculations performed using other more convenient formalisms (this reminds us of the story of Niels Bohr and the horseshoe.)

The formalism used is as said before completely algebraic devoid of any geometric insight. All symmetries are global since the action is fully gauge fixed. It is mainly useful to identify symmetries but also to discuss quantum perturbative behaviour. Hence it is important for a discussion about the convergence of the perturbation theory which we know works better for both pure gravity and the maximally supersymmetric one than expected. This is something to be studied now.

Having established the symmetry in four dimensions of spacetime it will also be important to go back to a geometric approach and find it there. This is usually a very difficult step, which is another drawback of the light-cone gauge formalism, but of course not impossible. Such a description would then reveal the true geometric background of the extended symmetry.

Our analysis raises the question of whether we actually know all the symmetries present in the field theories we work with. We know that Yang-Mills theory and gravity, both with and without supersymmetry, and particularly their maximally supersymmetric versions display remarkable quantum properties. We believe that we have taken a small step towards showing that there are symmetries beyond those we normally associate with these theories. We are very used, for good reasons, to working with covariant formalisms but what is the way forward when any new or hidden symmetries are only visible in non-covariant formulations or in spacetimes augmented with many extra coordinates? Our light-cone gauge formalism is democratic in the sense that all the symmetries are non-linearly implemented. This allows us, together with field redefinitions which are natural to perform in the functional integral over the action, to look for field representations which are particularly suited to these extra symmetries. We believe that there is room for further surprises.

\section{Acknowledgments}

We thank Chris Hull for valuable discussions. The work of SA is partially supported by a DST-SERB grant (EMR/2014/000687). SM acknowledges support from a CSIR 
NET fellowship. LB wishes to acknowledge Aspen Physics Center where part of his work was done.

Open Access. This article is distributed under the terms of the Creative Commons Attribution License (CC-BY 4.0), which permits any use, distribution and reproduction in any medium, provided the original author(s) and source are credited.

\section{References}

[1] Z. Bern et al., Amplitudes and ultraviolet behavior of $N=8$ supergravity, Fortsch. Phys. 59 (2011) 561 [arXiv: 1103.1848] [INSPIRE].

[2] G. Bossard, C. Hillmann and H. Nicolai, $E_{7(7)}$ symmetry in perturbatively quantised $N=8$ supergravity, JHEP 12 (2010) 052 [arXiv:1007.5472] [INSPIRE].

[3] E. Cremmer and B. Julia, The SO(8) supergravity, Nucl. Phys. B 159 (1979) 141 [inSPIRE].

[4] L. Brink, S.-S. Kim and P. Ramond, $E_{7(7)}$ on the light cone, JHEP 06 (2008) 034 [arXiv:0801.2993] [INSPIRE].

[5] S. Ananth, L. Brink and S. Majumdar, Exceptional versus superPoincaré algebra as the defining symmetry of maximal supergravity, JHEP 03 (2016) 051 [arXiv:1601.02836] [INSPIRE].

[6] S. Ananth, L. Brink and S. Majumdar, $E_{8}$ in $\mathcal{N}=8$ supergravity in four dimensions, JHEP 01 (2018) 024 [arXiv:1711.09110] [INSPIRE].

[7] S. Ananth, L. Brink, R. Heise and H.G. Svendsen, The $N=8$ supergravity hamiltonian as a quadratic form, Nucl. Phys. B 753 (2006) 195 [hep-th/0607019] [INSPIRE].

[8] S. Ananth, L. Brink and M. Mali, Yang-Mills theories and quadratic forms, JHEP 08 (2015) 153 [arXiv: 1507.01068] [INSPIRE].

[9] S. Ananth et al., Gravitation and quadratic forms, JHEP 03 (2017) 169 [arXiv:1702.06261] [INSPIRE].

[10] S. Ananth, S. Kovacs and S. Parikh, A manifestly MHV Lagrangian for $N=4$ Yang-Mills, JHEP 05 (2011) 051 [arXiv: 1101.3540] [INSPIRE].

[11] S. Ananth and S. Theisen, KLT relations from the Einstein-Hilbert Lagrangian, Phys. Lett. B 652 (2007) 128 [arXiv:0706.1778] [INSPIRE].

[12] J. Ehlers, Konstruktionen und Charakterisierung von Losungen der Einsteinschen Gravitationsfeldgleichungen, Dissertation, Hamburg University, Hamburg, Germany (1957).

[13] J. Scherk and J.H. Schwarz, Gravitation in the light-cone gauge, Gen. Rel. Grav. 6 (1975) 537 [INSPIRE].

[14] I. Bengtsson, M. Cederwall and O. Lindgren, Light cone actions for gravity and higher spins: some further result, Goeteborg University 83-55 (83,REC.APR.84) (1984).

[15] S. Ananth, The quintic interaction vertex in light-cone gravity, Phys. Lett. B 664 (2008) 219 [arXiv:0803.1494] [INSPIRE].

[16] L. Brink, O. Lindgren and B.E.W. Nilsson, $N=4$ Yang-Mills theory on the light cone, Nucl. Phys. B 212 (1983) 401 [INSPIRE].

[17] L. Brink, S.-S. Kim and P. Ramond, E $E_{8(8)}$ in light cone superspace, JHEP 07 (2008) 113 [arXiv: 0804.4300] [INSPIRE]. 